\title{
Impact of intermittent preventive treatment with sulphadoxine-pyrimethamine targeting the transmission season on the incidence of clinical malaria in children in Mali
}

\author{
Alassane Dicko*1,2, Issaka Sagara ${ }^{1}$, Mahamadou S Sissoko ${ }^{1}$, \\ Ousmane Guindo ${ }^{1}$, Abdoulbaki I Diallo ${ }^{1}$, Mamady Kone ${ }^{1}$, \\ Ousmane B Toure ${ }^{1}$, Massambou Sacko ${ }^{2}$ and Ogobara K Doumbo ${ }^{1}$
}

\begin{abstract}
Address: ${ }^{1}$ Malaria Research and Training Center, Departments of Epidemiology of Parasitic Diseases, Faculty of Medicine Pharmacy and Dentistry, University of Bamako, P.O. Box 1805, Bamako, Mali and ${ }^{2}$ Department of Public Health, Faculty of Medicine Pharmacy and Dentistry, University of Bamako, P.O. Box 1805, Bamako, Mali

Email: Alassane Dicko* - adicko@mrtcbko.org; Issaka Sagara - isagara@mrtcbko.org; Mahamadou S Sissoko - mssissoko@mrtcbko.org; Ousmane Guindo - guindoous@mrtcbko.org; Abdoulbaki I Diallo - abdoulbaki@mrtcbko.org; Mamady Kone - mamadi@mrtcbko.org; Ousmane B Toure - obtoure@mrtcbko.org; Massambou Sacko - sackom@ml.afro.who.int; Ogobara K Doumbo - okd@mrtcbko.org

* Corresponding author
\end{abstract}

Published: 8 July 2008

Malaria Journal 2008, 7:123 doi:10.1186/1475-2875-7-123
Received: 15 March 2008

Accepted: 8 July 2008

This article is available from: http://www.malariajournal.com/content/7/I/I23

(c) 2008 Dicko et al; licensee BioMed Central Ltd.

This is an Open Access article distributed under the terms of the Creative Commons Attribution License (http://creativecommons.org/licenses/by/2.0), which permits unrestricted use, distribution, and reproduction in any medium, provided the original work is properly cited.

\begin{abstract}
Background: Recent studies have shown that intermittent preventive malaria treatment (IPT) in infants in areas of stable malaria transmission reduces malaria and severe anaemia incidence. However in most areas malaria morbidity and mortality remain high in older children.

Methods: To evaluate the effect of seasonal IPT with sulphadoxine pyrimethamine (SP) on incidence of malaria disease in area of seasonal transmission, 262 children 6 months- 10 years in Kambila, Mali were randomized to receive either IPT with SP twice at eight weeks interval or no IPT during the transmission season of 2002 and were followed up for 12 months. Subjects were also followed during the subsequent transmission season in 2003 to assess possible rebound effect. Clinical malaria cases were treated with SP and followed to assess the in vivo response during both periods.

Results: The incidence rate of malaria disease per 1,000 person-months during the first 12 months was 3.2 episodes in the treatment group vs. 5.8 episodes in the control group with age-adjusted Protective Efficacy (PE) of $42.5 \%$; [95\% Cl $28.6 \%-53.8 \%$ ]. When the first 16 weeks of follow up is considered ageadjusted PE was $67.5 \%$ [ $95 \% \mathrm{Cl} 55.3 \%-76.6 \%]$. During the subsequent transmission season, the incidence of clinical malaria per 1000 persons-days was similar between the two groups (23.0 vs 21.5 episodes, ageadjusted IRR $=1.07[95 \% \mathrm{Cl}, 0.90-1.27])$. No significant difference was detected in in vivo response between the groups during both periods.

Conclusion: Two malaria intermittent treatments targeting the peak transmission season reduced the annual incidence rate of clinical malaria by $42.5 \%$ in an area with intense seasonal transmission. This simple strategy is likely to be one of the most effectives in reducing malaria burden in such areas.
\end{abstract}

Trial Registration: Clinicaltrials.gov NCT00623I55 


\section{Background}

Malaria is one of the most common infectious diseases in the world. It is estimated that malaria causes between 300 and 500 million clinical cases and 700,000 to 1.6 million deaths each year with $94 \%$ of deaths occurring in subSaharan Africa $[1,2]$.

In Mali, malaria is the leading cause of mortality and morbidity in the general population [3]. Like in many other Sahelian west African countries, malaria transmission is highly seasonal occurring during the rainy season, which varies from three to six months. It has been shown that more than $80 \%$ of malaria cases occurred during five months of the transmission season in the north savanna area of Mali. In such conditions, a suitable control strategy implemented during this period may have the most impact on the reduction of disease burden.

In the absence of vaccines, early case management and the use of insecticide-impregnated material are the best strategies to control malaria. However, their implementation has been slow and difficult to achieve due to the overarching poverty and illiteracy of the population as well as the insufficiency and inaccessibility of health services in most endemic areas. Home- and self-treatment have been proposed as alternatives to the insufficiency and inaccessibility of the health services, but these strategies have also several disadvantages, including misdiagnoses, lack of compliance with drug regimens, use of inappropriate medicine, which could contribute to development of drug resistance and the non-recognition of the severity of symptoms $[4,5]$. Consequently in sub-Saharan Africa, the morbidity and mortality of malaria are increasing [6]. There is an urgent need for an easy and simple malaria control strategy.

Recent, randomized controlled trials conducted in areas of perennial malaria transmission have shown that intermittent preventive treatment (IPT) given at the time of childhood vaccinations reduced the incidence of the first episode of malaria and severe anaemia by more than $50 \%$ during the first year of life, without there being any rebound in the subsequent year $[7,8]$. However, in countries such as Mali, where malaria is highly seasonal and prevalent in older children, IPT in infants (IPTi) may not be the optimum way in which to use antimalarial drugs to prevent malaria. An alternative approach is to give intermittent preventive treatment to children at risk only during the rainy season [9]. The primary aim of this study was to evaluate the impact of two seasonal IPT (sIPT) with sulphadoxine-pyrimethamine (SP) given at eight weeks interval on the incidence of malaria disease in children of six months to 10 years of age in an area of seasonal transmission, in Kambila, Mali. Secondary aims were to assess the impact of this strategy on the in vivo response of Plas- modium falciparum to SP and the potential rebound effect of this strategy on the subsequent transmission season after its cessation.

\section{Study design and methods Study site}

The study was conducted in Kambila, a rural village of about 1,500 inhabitants located about $25 \mathrm{~km}$ from Bamako, the capital city of Mali. Plasmodium falciparum is hyperendemic with parasitaemia prevalence rates ranging from $40-50 \%$ in the dry season (November - May) and $70-85 \%$ in the rainy season (June - October). The bed net coverage in the area at the time of the survey was less than $5 \%$. The village was chosen to represent an area with four to five months of seasonal malaria transmission. SP was at the time, the second-line antimalarial drug in Mali, and was recommended for chloroquine failures. In 1995, a previous study found SP efficacy to be greater than $99 \%$ in the neighbouring area [10].

\section{Study participants and design}

The study was an open randomized controlled clinical trial. After a census of the village population, subjects in the target age group were screened for inclusion and exclusion criteria. Subjects who met inclusion and exclusion criteria were randomized either to receive two intermittent preventive treatments with standard recommended treatment doses of SP or no intermittent preventive treatment. Randomization codes were computer generated using simple randomization technique and treatment allocations were provided within sealed opaque envelopes. The first treatment was given at the beginning of the transmission season in July 2002 and the second treatment eight weeks later in August 2002. Inclusion criteria for the study included: 1) parental or other legal guardian consent; 2) age six months to 10 years; 3) having no chronic illness or symptomatic malaria; 4) agreeing to seek initial medical care for all medical illness in the study clinic during the entire study period; 5) having no plan to travel for a long time during the study period. Specific exclusion criteria included children with a history of allergy to sulpha drugs or SP.

\section{Follow-up}

Subjects from both groups were actively and passively followed during the study periods. The active follow-up was done weekly and consisted of: 1) questioning the subjects or parent for presence within the two past days of symptoms consistent with malaria including fever, headaches, body aches, malaise, diarrhoea, vomiting, and abdominal pain; 2) recording the axillary temperature; 3 ) examining conjunctivae and palms for pallor indicating a profound anaemia. The passive follow-up was done through continuous availability of study clinicians to evaluate any medical complaint at anytime during the entire study period. 
Any subject, who complains of symptom consistent with malaria, with an axillary temperature greater or equal to $37.5^{\circ} \mathrm{C}$, or with profound anaemia or jaundice, was given a complete medical exam and laboratory assessment including malaria smear and haemoglobin or hematocrit measurement. The criteria for discontinuing further follow-up for a volunteer were: 1) consent withdrawal, 2) volunteer missed three consecutives follow-up visits, 3) assessment of the study physician as to whether being in the study was in the best interest of the volunteer.

\section{Treatment}

Subjects who were diagnosed with uncomplicated malaria were treated immediately with standard doses of SP, and those who were diagnosed with severe malaria were treated with quinine according to the guidelines of National Malaria Control Programme. They remained in the study so that multiple episodes of malaria disease could be detected over the course of the study period. In vivo response to SP was assessed using the WHO 2003 protocol [11] on uncomplicated malaria cases. Clinical assessments were made on days 1, 2, 3, 7, 14, 21 and 28 following treatment with SP, and blood smears were obtained (by finger prick) on days 3, 7, 14, 21 and 28 to determine the in vivo SP treatment failure. At the end of every clinic visit, subjects and their parent/legal guardian were encouraged to return immediately to the study clinic and/or their primary health care system should any other new symptoms appear.

Standard recommended treatment doses of SP (Fansidar ${ }^{\oplus}$, F. Hoffman-La Roche Ltd, Basel, Switzerland) was given for IPT and for treatment of episodes of uncomplicated malaria ( $1 / 4$ tablet per $5 \mathrm{~kg}$ wt for age $\leq 12$ years). All subjects were observed for at least 60 minutes for vomiting. If vomiting occurred within 30 minutes, the full dose was repeated and if it occurred within 60 minutes, $1 / 2$ of the dose was repeated. Cases of SP failure, and cases of malaria disease occurring within two weeks of a periodic treatment in the IPT group, were treated with chloroquine at $25 \mathrm{mg} / \mathrm{kg}$ in three days. Severe and complicated malaria cases were treated with quinine $8 \mathrm{mg} / \mathrm{kg}$ three times a day for five days. Serious adverse events were monitored during the duration of the study.

\section{Laboratory methods}

Haematocrit was determined using microhaematocrit reading device after centrifugation (IECMicro-MB centrifuge) and haemoglobin concentration was determined using a portable analyzer (Hemocue, Lake Forest, CA). Parasitaemia was assessed by counting the number of asexual $P$. falciparum parasites on Giemsa-stained thick blood films until 300 leukocytes were observed by microscopists unaware of the treatment group of the subject. Parasite densities were converted assuming 7,500 leuko- cytes $/ \mu$ l. Routine quality control was performed on $10 \%$ of the slides, with a second microscopist re-examining the blood films while blinded to the previously recorded result. Differences in parasitaemia of more than $10 \%$ were resolved by an expert microscopist.

\section{Study endpoints}

The primary end point of the study was the incidence rate of clinical malaria, defined as uncomplicated or severe malaria for the assessment of protective efficacy and the rebound effect. Uncomplicated malaria was defined as signs or symptoms consistent with malaria either leading to treatment-seeking behaviour or reported during weekly follow-up visits accompanied by any level of parasitaemia. These signs and symptoms included fever at the time of evaluation (axillary temperature $\geq 37.5^{\circ} \mathrm{C}$ by digital thermometer), profound anaemia (conjunctival or palmar pallor), jaundice, report of fever within the last two days, lassitude, headache, body aches, diarrhoea, or abdominal pain. Severe malaria was defined according to the most recent WHO protocols: severe anaemia (defined as haemoglobin $<5 \mathrm{~g} / \mathrm{dL}$ ); parasitemia $>10 \%$ or $500,000 /$ $\mathrm{mm}^{3}$, prostration, respiratory distress, bleeding, recent repeated seizures, coma or obtundation, inability to drink, or persistent vomiting. The secondary endpoint was the non PCR corrected adequate clinical and parasitological response (ACPR) for the in vivo response of $P$. falciparum to SP. ACPR of $P$. falciparum to SP was defined according the WHO 2003 protocol [11] a protocol already available in 2002.

\section{Sample size}

The sample size was computed using the primary end point which was the incidence rate of malaria disease. Using normal approximation of square root transformation of Poisson formula, 96 persons-years of follow-up in each group were needed in order to detect a reduction of $40 \%$ in the incidence malaria disease, with $90 \%$ power and $95 \%$ confidence level, based on the estimated incidence of 1.2 episodes per person-year in the non-treatment group. To account for loss to follow up a total of 262 subjects ( 131 for each group) were enrolled. Using equivalency study of interventions method a total of 120 in vivo tests per arm will give more than $80 \%$ power for a maximum difference of $7 \%$ in ACPR between the two arms assuming ACPR of 95\%.

\section{Statistical analysis}

Intention to treat analysis was used. The person-time method was used to calculate the incidence rate of malaria disease and to account for variable length of follow-up. The incidence rates were calculated as number of malaria episodes divided by the number of person days of followup at risk in each group. The incidence rates in the two groups were compared adjusted for age as continuous var- 
iable, using Poisson regression models in overall and for each age category ( $<5$ years and $>=5$ years). Subjects were not considered at risk for 28 days after a treatment for malaria disease. Protective efficacy of two intermittent treatments with SP was computed as 1 minus the incidence rate ratio of malaria disease over the first 52 weeks. This was also computed for the first 16 weeks to allow comparison with other studies. Proportions of subjects with ACPR using WHO latest protocol [11] in the two groups were compared during the first year and the subsequent transmission season $\left(53^{\text {rd }}\right.$ to $75^{\text {th }}$ week in December 2003) using Pearson chi square or Fisher exact tests as appropriate. Parasite densities were log transformed and geometric means parasite densities were compared using Student $t$ test. Data were entered and verified using MS Access and then exported to Stata (StataCorp, College Station, Texas, US) for analysis.

\section{Ethical considerations}

The protocol was approved by the Ethical Committee of Faculty of Medicine, Pharmacy and Dentistry of the University of Bamako, Mali. Community permission and written individual consent from parent or legal guardian were obtained before inclusion in the study.

\section{Role of the funding source}

The study was funded by the UNDP/World Bank/WHO Special Programme for Research and Training in Tropical Diseases (TDR), re-entry grant ID AI0828. Additional funds came from the President Clinton donation to MRTC through NIAID/NIH. The two institutions had no role in the design conduct analysis and reporting of the study. The corresponding author had full access to all the data and had final responsibility for the decision to submit for publication.

\section{Results}

\section{Characteristics of the study participants}

A total of 262 subjects were enrolled in this study (131 in each arm). Baseline characteristics are summarized in Table 1. There was no significant difference between the

Table I: Baseline characteristics of the study participants

\begin{tabular}{lccc}
\hline & $\begin{array}{c}\text { sIPT }+ \\
(n=131)\end{array}$ & $\begin{array}{c}\text { sIPT- } \\
(n=13 \mid)\end{array}$ & $P$ \\
\hline $\begin{array}{l}\text { Mean age in years (SD) } \\
\text { Sex (\% of male) }\end{array}$ & $5.6(2.9)$ & $5.1(2.8)$ & n.s. \\
Parasite prevalence (\%) & 50.4 & 58.0 & n.s. \\
Lost to follow up first year (\%) & 33.6 & 37.4 & n.s. \\
$\quad$ Week I to 52 & 11.4 & 11.4 & n.s. \\
Week 53 to 75 & 5.3 & 3.8 & n.s.
\end{tabular}

$\mathrm{SD}=$ standard deviation

sIPT - = control group

sIPT + = treatment group

n.s. not significant two groups at baseline in term of age, gender and malaria parasite prevalence.

\section{Loss to follow-up}

Of the 262 subjects enrolled, 30 subjects (11.4\%) did not complete one year follow up and were equally distributed between the two groups (15 in each group). An additional 12 subjects $(4.6 \%)$ did not complete the extended followup 7 in treatment group and 5 in the control group (Figure 1). Overall proportion of subjects who did not complete the follow up was similar between the two groups $(16.8 \%$ versus $15.3 \%, \mathrm{p}=0.73$ ). The reasons for loss to follow-up during the study were: migration to another location $(\mathrm{n}=$ $32)$, missing more three consecutive weekly visits $(n=6)$, consent withdrawal $(n=3)$ and death from severe malaria $(\mathrm{n}=1)$.

\section{Incidence rate of malaria disease and efficacy of sIPT after 52 weeks of follow up}

The incidence rate and protective efficacy after 52 weeks of follow up are presented in Table 2. Overall 352 episodes of malaria disease occurred, 221 in the control group and 130 in the sIPTc group. The incidence rates per 1000 person-days at risk for the malaria disease were 5.8 and 3.2 in the control and treatment groups respectively, giving a protective efficacy of $44.8 \%$ (95\% CI $31.5 \%$ to $55.6 \%$ ). After adjustment for age, the protective efficacy was $42.5 \%$ (95\% CI 28.6 to $53.8 \%$.) (Table 2). When the analysis was stratified by age categories, the incidence rate of malaria disease was 7.3 and 3.9 episodes per 1000 person-days in control and treatment groups respectively with an ageadjusted PE of $46.6 \%$ (95\% CI $28.0 \%$ to $60.4 \%$ ) in children less than five years of age. In children five years and above, the incidence rate of malaria disease were 4.5 and 2.7 episodes per 1000 person-days respectively in the control and treatment group. Age adjusted PE in this age group was $35.9 \%$ (95\% CI $11.8 \%$ to $53.4 \%$ ).

Five cases of severe malaria (two cases of cerebral malaria, two cases of hyperparasitaemia, and one case of severe anaemia) occurred during the first 12 months of the follow-up, all in the control group giving an incidence rate of 0.048 episodes per 1000 persons-days at risk and a cumulative incidence of $3.9 \%$ in this group.

\section{Incidence rate of malaria disease and efficacy of sIPT after 16 weeks of follow-up}

The incidence rate and protective efficacy after the first 16 weeks of follow-up are presented in Table 3. A total of 200 malaria disease episodes occurred during the first 16 weeks of follow-up, 145 in the control group and 55 in the group who received sIPT. The age adjusted PE in this period was $67.5 \%$ (95\% CI 55.3\% to $76.6 \%$ ) and was not significantly different in subjects of less than five years compared to those aged of five years and above. 


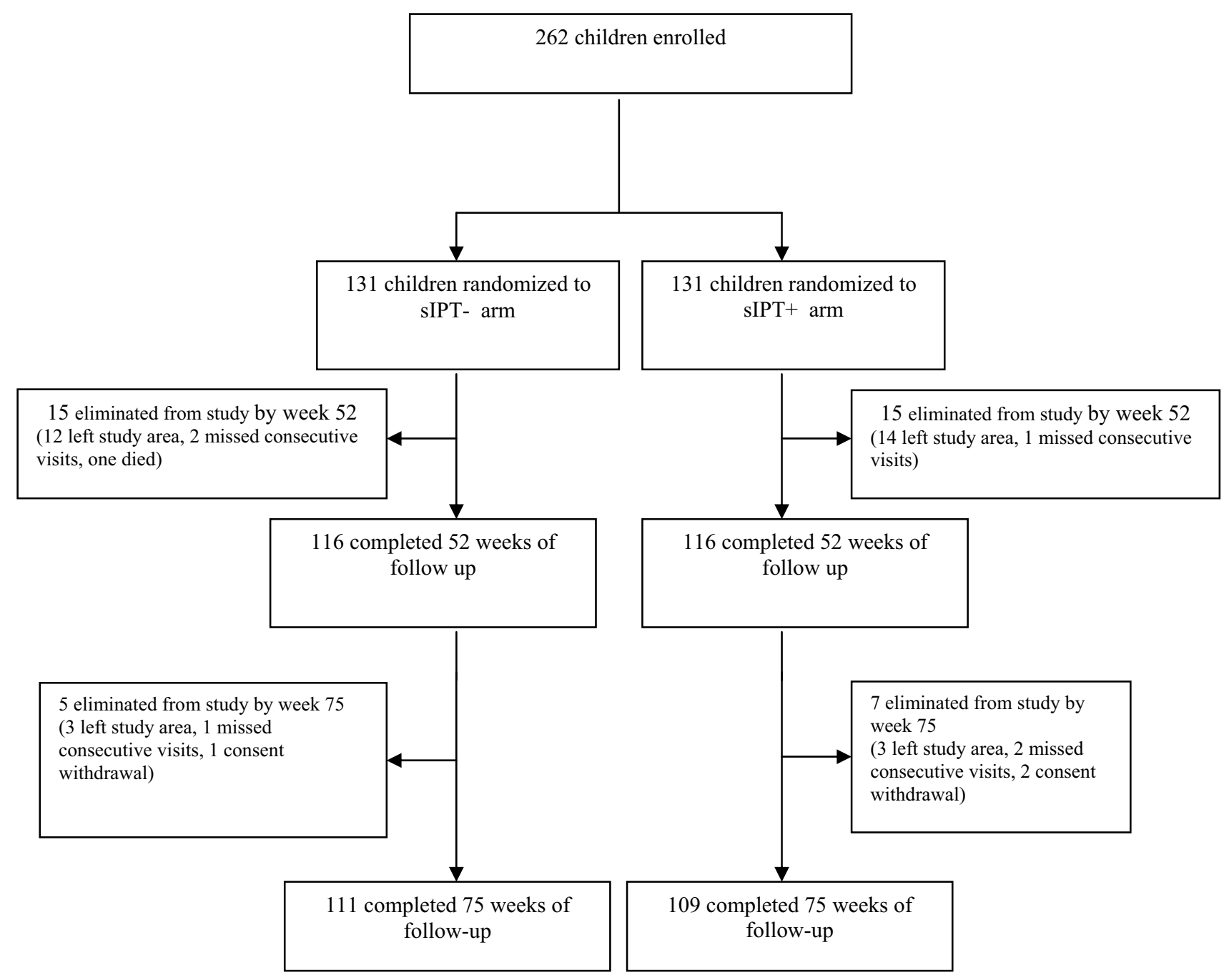

Figure I

Flow Chart.

Table 2: Incidence rate of clinical malaria and protective efficacy after 52 weeks of follow-up per treatment group and age category

\begin{tabular}{|c|c|c|c|c|c|c|}
\hline & \multicolumn{2}{|c|}{$<5$ years } & \multicolumn{2}{|c|}{$5-10$ years } & \multicolumn{2}{|c|}{ All } \\
\hline & sIPT- & slPT + & sIPT- & slPT + & sIPT- & sIPT + \\
\hline Number of malaria episodes & 127 & 65 & 94 & 65 & 221 & 130 \\
\hline $\begin{array}{l}\text { Person-days (-years) of follow } \\
\text { up }\end{array}$ & $|73| \mid(47.6)$ & $16590(45.6)$ & $20799(57.1)$ & $24025(66.0)$ & $38110(104.7)$ & $40615(111.6)$ \\
\hline $\begin{array}{l}\text { Incidence rate malaria disease } \\
\text { per } 1000 \text { person-days (-years) } \\
\text { of follow up }\end{array}$ & $7.3(2.67)$ & $3.9(1.43)$ & $4.5(1.65)$ & $2.7(0.98)$ & $5.8(2.11)$ & $3.2(1.16)$ \\
\hline Unadjusted $\mathrm{PE}$ in \% $(95 \% \mathrm{Cl})$ & Reference group & $46.6(28.0-60.4$ & Reference group & $40.1(17.9-56.4)$ & Reference group & $44.8(31.5-55.6)$ \\
\hline Age adjusted $\mathrm{PE}$ in $\%(95 \% \mathrm{Cl})$ & Reference group & $46.6(28.0-60.4)$ & Reference group & $35.9(11.8-53.4)$ & Reference group & $42.5(28.6-53.8)$ \\
\hline
\end{tabular}

SIPT - = control group

sIPT + = treatment group

$\mathrm{PE}=$ Protective efficacy 
Table 3: Incidence rate of malaria disease and protective efficacy after 16 weeks of follow up per treatment group and age category

\begin{tabular}{|c|c|c|c|c|c|c|}
\hline & \multicolumn{2}{|c|}{$<5$ years } & \multicolumn{2}{|c|}{$5-10$ years } & \multicolumn{2}{|c|}{ All } \\
\hline & sIPT- & sIPT + & sIPT- & sIPT + & sIPT- & sIPT + \\
\hline Number of malaria episodes & 86 & 30 & 59 & 25 & 145 & 55 \\
\hline Person-days of follow up & 4797 & 5474 & 6207 & 7875 & 11004 & 13349 \\
\hline $\begin{array}{l}\text { Incidence rate malaria disease } \\
\text { per } 1000 \text { person-days of follow } \\
\text { up }\end{array}$ & 17.9 & 5.5 & 9.5 & 3.2 & 13.2 & 4.1 \\
\hline $\mathrm{PE}$ in \% $(95 \% \mathrm{Cl})$ & Reference group & $68.7(57.4-77.1)$ & Reference group & $66.6(46.7-79.1)$ & Reference group & $68.7(57.4-76.1)$ \\
\hline Age adjusted $\mathrm{PE}$ in $\%(95 \% \mathrm{Cl})$ & Reference group & $69.4(53.6-79.8)$ & Reference group & $63.4(4 \mid .5-77.1)$ & Reference group & $67.5(55.3-76.6)$ \\
\hline
\end{tabular}

sIPT - = control group

sIPT + = treatment group

$\mathrm{PE}=$ Protective efficacy

Incidence rate of malaria disease after cessation of sIPT during the next subsequent transmission season (weeks 53 to 75)

During the subsequent malaria transmission season in 2003, a total of 503 malaria episodes occurred, with 255 cases in the treatment group and 248 in the control group. The incidence rates of malaria disease were similar between the two groups 23.0 episodes per 1,000 personsdays in the treatment group and 21.5 episodes per 1,000 persons-days in the control group with age adjusted Incidence Rate Ration (IRR) $=1.07$ (95\% CI 0.90 to $1.27, \mathrm{p}=$ 0.46). During this extended follow up period two cases of severe malaria occurred, both type cerebral malaria and in the control group.

\section{In vivo response of $P$. falciparum to $S P$}

Of the 351 malaria episodes during the first year, valid in vivo tests were performed in 331 (94.3\%) with 119/130 $(91.5 \%)$ in the control group and 212/221 (95.9\%) in the sIPT group. Table 4 shows that non PCR corrected 28 days Adequate Clinical and Parasitological Response (ACPR) was similar in the two groups in overall $(92.9 \%$ in the control group vs. 94.1\% in the sIPT group) and when the analysis was stratified by age categories. Mean parasitaemia at day 0 was also similar between the two groups with geometric means parasitaemia per $\mu$ l of 11,480 (95\% CI $8,770$ to 15,027$)$ in the control group versus $9,478(95 \%$ CI 6,639 to 13,530 ) in the treatment group, $\mathrm{p}=0.39$.

During the extended surveillance following cessation of sIPT (53 ${ }^{\text {rd }}$ to $75^{\text {th }}$ week), valid in vivo tests were performed in 472 (93.8\%) of the 503 malaria episodes were treated with SP. Mean parasitaemia at day 0 was also similar between the two groups with geometric means parasitaemia per $\mu$ l of $8,191(95 \%$ CI of 6,367 to 10,538$)$ in the control group versus 9,903 (95\% CI 7,704 to 12,730$)$ in the treatment group, $\mathrm{p}=0.29$.

In vivo responses per treatment and follow up period are presented in Table 5. The ACPR were similar between the two groups ( $87.9 \%$ versus $89.6 \%$ ). The same similarities were found when the analysis was done in children less than five years of age or in those aged of five years and above.

Table 4: In vivo response of $P$. falciparum to SP during the first year of follow up group age categories and treatment groups

\begin{tabular}{|c|c|c|c|c|c|c|}
\hline \multirow[b]{2}{*}{ Outcome } & \multicolumn{2}{|c|}{$<5$ years } & \multicolumn{2}{|c|}{$5-10$ years } & \multicolumn{2}{|c|}{ All } \\
\hline & $\begin{array}{c}\text { sIPT- } \\
(\mathrm{n}=122)\end{array}$ & $\begin{array}{c}\text { sIPT + } \\
(n=58)\end{array}$ & $\begin{array}{c}\text { slPT- } \\
(n=90)\end{array}$ & $\begin{array}{c}\text { sIPT + } \\
(\mathrm{n}=6 \mathrm{I})\end{array}$ & $\begin{array}{c}\text { sIPT- } \\
(\mathrm{n}=2 \mid 2)\end{array}$ & $\begin{array}{c}\text { slPT + } \\
(\mathrm{n}=119)\end{array}$ \\
\hline ACPR & 93.4 & 93.1 & 92.2 & 95.1 & 92.9 & 94.1 \\
\hline ETF & 0.0 & 1.7 & 0.0 & 0 & 0.0 & 0.8 \\
\hline LCF & 0.0 & 0.0 & 0.0 & 0 & 0.0 & 0.0 \\
\hline LPF & 6.6 & 5.2 & 7.8 & 4.9 & 7.1 & 5.0 \\
\hline
\end{tabular}

sIPT - = control group

sIPT + = treatment group

$\mathrm{ACPR}=$ adequate clinical and parasitological response

$\mathrm{ETF}=$ early treatment failure

LPF = late parasitological failure 
Table 5: In vivo response of $P$. falciparum to SP during the extended period of follow up after cessation of sIPT implementation per age categories and treatment group

\begin{tabular}{|c|c|c|c|c|c|c|}
\hline \multirow[b]{2}{*}{ Outcome } & \multicolumn{2}{|c|}{$\mathrm{I}-4$ years } & \multicolumn{2}{|c|}{$5-11$ years } & \multicolumn{2}{|c|}{ All } \\
\hline & $\begin{array}{c}\text { slPT- } \\
(n=102)\end{array}$ & $\begin{array}{c}\text { sIPT + } \\
(n=104)\end{array}$ & $\begin{array}{c}\text { slPT- } \\
(n=130)\end{array}$ & $\begin{array}{c}\text { sIPT + } \\
(n=136)\end{array}$ & $\begin{array}{c}\text { sIPT- } \\
(n=232)\end{array}$ & $\begin{array}{c}\text { sIPT + } \\
(n=240)\end{array}$ \\
\hline ACPR & 87.3 & 84.6 & 88.5 & 93.4 & 87.9 & 89.6 \\
\hline ETF & 0.0 & 1.0 & 1.5 & 0.7 & 0.9 & 0.8 \\
\hline $\mathrm{LCF}$ & 1.0 & 0.0 & 0.0 & 0.0 & 0.4 & 0.0 \\
\hline LPF & 11.8 & 14.4 & 10.0 & 5.9 & 10.8 & 9.6 \\
\hline
\end{tabular}

sIPT - = control group

sIPT + = treatment group

$\mathrm{ACPR}=$ adequate clinical and parasitological response

ETF = early treatment failure

LPF = late parasitological failure

Overall there was a reduction in the efficacy of SP during the extended follow-up compared to the first year of the follow up, especially in children less than five years of age.

\section{Safety}

No SP related serious adverse event was recorded during the study period. No subject was withdrawn because of allergy to SP.

\section{Discussion}

This study assessed the impact of two doses of intermittent preventive treatment with SP at 8-week intervals in children of six months to 10 years of age targeting the transmission season in Kambila, Mali and found a reduction of $42.5 \%$ in annual incidence of clinical malaria. When SP plus single dose of artesunate was given at monthly intervals in an area with seasonal malaria transmission, Cisse et al [12] in Senegal found a reduction of $86 \%$ in incidence of clinical malaria children less than five years of age over 13 weeks period. This higher efficacy can be explained by the shorter time interval between treatments (four weeks instead of eight weeks), the number of intermittent treatments (three instead of two) and the shorter duration of the follow up (13 instead 52 weeks) [12]. Furthermore when the analysis of the efficacy of the two doses of intermittent preventive treatment with SP at 8 -week intervals was limited to the first 16 weeks of follow up, the protective efficacy was $69.4 \%$ in children aged less than five years. Although the incidence rate of clinical malaria was higher in children less than five years of age compared to those of five years and above, the differences in efficacy of the strategy were not significantly different suggesting that IPT is also appropriate for older children.

While several studies have assessed the impact of IPT in pregnant women and infants, few studies have assessed the impact of this strategy in children. Several studies have shown that intermittent treatment given at 3, 6, 9 months of age reduced the incidence of clinical episodes by 20 to
$60 \%$ with strong variations according to transmission duration and the seasonality $[7,8,13,14]$. In areas of seasonal malaria transmission, the burden of malaria remains high in older children in addition to infants [15$20]$. Furthermore, more than $80 \%$ of the malaria cases in non-irrigated areas occurred during the 4-6 months of the transmission season [21], suggesting that sIPT is an appropriate preventive strategy for malaria control.

Unlike the use of other malaria control strategies such as use of insecticide-impregnated material that require daily implementation, this strategy requires only a twice-yearly treatment while offering significant protection against malaria. This simple strategy can be delivered through schools and/or local and national campaigns. Despite the current evidence and recommendation for use of insecticide-treated nets (ITN), this strategy remains largely under-utilized in Mali and many parts of Africa [22-24]. Increasingly, as efforts are being made to expand coverage of ITN it will be interesting to assess the efficacy of sIPT in children in the context of wide use of ITN.

The incidence rates of malaria disease (including severe malaria) reported in this randomized control trial were similar to those reported in previous studies in Mali $[18,25]$. This suggests an unbiased measure of the outcome despite the fact that the study was not a placebo controlled one, and that similar efficacy can be achieved by sIPTc in these areas.

There was no significant difference in SP efficacy in vivo between the treatment and control groups during the two periods of follow up using the WHO 2003 protocol for assessing the in vivo efficacy of antimalarials [11]. However there was a reduction in SP efficacy during the extended follow up period compared to the first year of follow up, especially among children less than five years of age. It is difficult to conclude if this reduction in SP in vivo efficacy was due to the sIPTc or was rather the 
expected decrease in efficacy due to the use of SP for treatment of malaria episodes in this population. In this study, uncomplicated malaria cases in both arms were treated with SP $\left(2^{\text {nd }}\right.$ line malaria treatment in Mali at the time of the study). While the higher efficacy and the longer prophylactic effect of SP compared to choloroquine (the first line treatment at the time of the study), provide more benefice for the study participants, this may contribute to the lack of difference in in vivo response between the two arms and the trend towards a lower efficacy during the extended follow up period.

Cisse et al [12] have found variation in frequency of molecular markers to SP resistance according to the season, without clear link with the intermittent preventive treatment. Results of studies undertaken by the IPTi consortium will bring more insight on the impact of IPT on drug resistance and whether this is an acceptable price to pay for the substantive benefit of this strategy and whether the combination with artesunate would be able to reduce the risk of increased resistance.

As shown in other studies $[7,8,12]$ the cessation of the strategy was not associated with an increase of malaria disease during the subsequent transmission season. However in all these studies, IPT was given for a short time usually one year. It would be interesting to assess whether seasonal IPT in children given over longer periods impairs immunity to malaria.

In conclusion two malaria intermittent treatments targeting the peak of the transmission season have substantially reduced the incidence of clinical malaria in this area with intense seasonal transmission without a rebound effect after cessation. The strategy is simple and is likely to be very effective in reducing malaria burden in areas with seasonal malaria transmission.

\section{Completing interests}

The authors declare that they have no competing interests.

\section{Authors' contributions}

AD was the principal investigator of the study. He drafted the protocol, oversees the conduct of the study and contributed to the data analysis and interpretation. IS monitored the data quality of the study and contributed to the data analysis. MSS assisted with the implementation and coordination of the field activities. Data collection in the field data was done by OG, AID and MK. OBT assisted with the data management. MS contributed in overseeing the study. OKD contributed in the design of the study and in overseeing the data collection, analysis and interpretation. The manuscript was drafted by $\mathrm{AD}$ and all the authors contributed to revision and approved the final version.

\section{Acknowledgements}

We thank the children who participate into the study, their parents and the population of village of Kambila. We are also grateful to Dr. Sibylle Kristensen and Ms. Heather White for their critical reading of the manuscript. Results were presented in part at the American Society of Tropical Medicine and Hygiene 52nd Annual Meeting, December, 2004.

\section{References}

I. World Health Organization: Burden of disease in DALYs by cause sex and mortality stratum in the WHO regions, estimates for 200I. World Health Report 2002: Reducing risks, Promoting Healthy life 2002:192-197 [http://www.who.int/whr/2002/ whr2002 annex3.pdf]. Geneva: WHO

2. Adjuik M, Smith T, Clark S, Todd J, Garrib A, Kinfu Y, Kahn K, Mola M, Ashraf A, Masanja H, Adazu K, Sacarlal J, Alam N, Marra A, Gbangou A, Mwageni E, Binka F: Cause-specific mortality rates in sub-Saharan Africa and Bangladesh. Bull World Health Organ 2006, 84: $181-188$.

3. Duflo B, Balique H, Ranque P, Diallo AN, Brucker G, Alavi H, Prescott $\mathrm{N}$ : [Estimation of the impact of the principal diseases in rural Mali]. Rev Epidemiol Sante Publique 1986, 34:405-4I8.

4. Théra MA, D'Alessandro U, Thiéro M, Ouedraogo A, Packou J, Deida OAS, Fané M, Ade G, Alvez F, Doumbo O: Child malaria treatment practices among mothers in the district of Yanfolila, Sikasso Region, Mali. Trop Med Int Health 2000, 5:876-88I.

5. Health Systems and Operational Research Focus Group Report: International Conference on malaria in Africa: Challenges and opportunities for cooperation. Final Report, Dakar 1997.

6. Trape JF: The public health impact of chloroquine resistance in Africa. Am J Trop Med Hyg 200I, 64(1-2 Suppl): I2-I7.

7. Schellenberg D, Menendez C, Kahigwa E, Aponte J, Vidal J, Tanner M, Mshinda $\mathrm{H}$, Alonso $\mathrm{P}$ : Intermittent treatment for malaria and anaemia control at time of routine vaccinations in Tanzanian infants: a randomised, placebo-controlled trial. Lancet 2001, 357:147|-|477.

8. Massaga JJ, Kitua AY, Lemnge MM, Akida JA, Malle LN, Ronn AM, Theander TG, Bygbjerg IC: Effect of intermittent treatment with amodiaquine on anaemia and malarial fevers in infants in Tanzania: a randomised placebo-controlled trial. Lancet 2003, 36 I: | $853-1860$.

9. Greenwood BM: The use of anti-malaria drugs to prevent malaria in the population of malaria-endemic areas. Am J Trop Med Hyg 2004, 70:1-7.

10. Diourté Y, Djimde A, Doumbo O, Sagara I, Coulibaly Y, Dicko A, Diallo M, Diakite M, Cortese JF, Plowe CV: Pyrimethamine sulfadoxine efficacity and selection for mutations in Plasmodium falciparum dihydrofolate reductase and dihydropteroate synthase in Mali. Am J Trop Med Hyg 1999, 60:475-478.

II. World Health Organization: Assessment of therapeutic efficacy of antimalarial drugs for uncomplicated falciparum malaria. WHO/HTM/RBM/ 2003.50 [http://www.who.int/malaria/includes en/ whomalariapublications 19982004.htm\#2003].

12. Cisse B, Sokhna C, Boulanger D, Milet J, Ba el H, Richardson K, Hallett R, Sutherland C, Simondon K, Simondon F, Alexander N, Gaye O, Targett, Lines J, Greenwood B, Trape JF: Seasonal intermittent preventive treatment with artesunate and sulfadoxinepyrimethamine for prevention of malaria in Senegalese children: a randomised, placebo-controlled, double-blind trial. Lancet 2006, 367:659-667.

13. Chandramohan D, Owusu-Agyei S, Carneiro I, Awine T, AmponsaAchiano K, Mensah N, Jaffar S, Baiden R, Hodgson A, Binka F, Greenwood $\mathrm{B}$ : Cluster randomised trial of intermittent preventive treatment for malaria in infants in area of high, seasonal transmission in Ghana. BMJ 2005, 331:727-733.

14. Macete E, Aide P, Aponte JJ, Sanz S, Mandomando I, Espasa M, Sigauque B, Dobano C, Mabunda S, Dgedge M, Alonso P, Menendez C: Intermittent preventive treatment for malaria control administered at the time of routine vaccinations in mozambican infants: a randomized, placebo-controlled trial. J Infect Dis 2006, 194:276-285.

15. Trape JF, Rogier C: Combating malaria morbidity and mortality by reducing transmission. Parasitol Today 1996, I 2:236-240.

16. Coulibaly D, Diallo DA, Thera MA, Dicko A, Guindo AB, Kone AK Cissoko Y, Coulibaly S, Djimde A, Lyke K, Doumbo O, Plowe CV: 
Impact of preseason treatment on incidence of falciparum malaria and parasite density at a site for testing malaria vaccines in Bandiagara, Mali. Am J Trop Med Hyg 2002, 67:604-610.

17. Lalloo DG, Olukoya P, Olliaro P: Malaria in adolescence: burden of disease, consequences, and opportunities for intervention. Lancet Infect Dis 2006, 6:780-793.

18. Dicko A, Sagara I, Diemert DJ, Sogoba M, Niambélé MB, Dao A, Dolo G, Yalcouyé D, Diallo DA, Saul A, Miller LH, Touré YT, Klion A, Doumbo OK: Year-to-year variation in the age-specific incidence of malaria in two potential vaccine testing sites in Mali with different levels of malaria transmission intensity. Am J Trop Med Hyg 2007, 77: 1028-1033.

19. Greenwood B: Intermittent preventive antimalarial treatment in infants. Clin Infect Dis 2007, 45:26-28.

20. Chandramohan D, Webster J, Smith L, Awine T, Owusu-Agyei S, Carneiro I: Is the Expanded Programme on Immunisation the most appropriate delivery system for intermittent preventive treatment of malaria in West Africa. Trop Med Int Health 2007, I 2:743-750.

21. Sissoko MS, Dicko A, Briet OJ, Sissoko M, Sagara I, Keita HD, Sogoba $M$, Rogier C, Toure YT, Doumbo OK: Malaria incidence in relation to rice cultivation in the irrigated Sahel of Mali. Acta Trop 2004, 89:161-170

22. Rhee M, Sissoko M, Perry S, Dicko A, McFarland W, Doumbo O Malaria prevention practices in Mopti region, Mali. East Afr Med J 2005, 82:396-402.

23. Miller JM, Korenromp EL, Nahlen BLW, Steketee R: Estimating the number of insecticide-treated nets required by African households to reach continent-wide malaria coverage targets. JAMA 2007, 297:224I-2250.

24. Hill J, Lines J, Rowland M: Insecticide-treated nets. Adv Parasitol 2006, 61:77-128.

25. Lyke KE, Dicko A, Kone A, Coulibaly D, Guindo A, Cissoko Y, Traore K, Plowe CV, Doumbo OK: Incidence of severe Plasmodium falciparum malaria as a primary endpoint for vaccine efficacy trials in Bandiagara, Mali. Vaccine 2004, 22:3169-3174.

Publish with Biomed Central and every scientist can read your work free of charge

"BioMed Central will be the most significant development for disseminating the results of biomedical research in our lifetime. "

Sir Paul Nurse, Cancer Research UK

Your research papers will be:

- available free of charge to the entire biomedical community

- peer reviewed and published immediately upon acceptance

- cited in PubMed and archived on PubMed Central

- yours - you keep the copyright

Submit your manuscript here:

http://www.biomedcentral.com/info/publishing_adv.asp
BioMedcentral 